\title{
Transcriptomic landscaping of core genes and pathways of mild and severe psoriasis vulgaris
}

\author{
SAUMYA CHOUDHARY $^{1,2}$, RISHIKA ANAND ${ }^{3}$, DIBYABHABA PRADHAN ${ }^{4}$, BANAJIT BASTIA BA, $^{2,5}$ \\ SHASHI NANDAR KUMAR ${ }^{5,6}$, HARPREET SINGH ${ }^{4}$, POONAM PURI $^{7}$, \\ GEORGE THOMAS $^{1}$ and ARUN KUMAR JAIN ${ }^{2,5}$
}

\begin{abstract}
${ }^{1}$ Department of Molecular and Cellular Engineering, Sam Higginbottom University of Agriculture, Technology and Sciences, Prayagraj (Allahabad), Uttar Pradesh 211007; ${ }^{2}$ Biomedical Informatics Centre, ICMR-National Institute of Pathology,

New Delhi 110029; ${ }^{3}$ Amity Institute of Biotechnology, Amity University, Noida Uttar Pradesh 201313;

${ }^{4}$ ICMR-AIIMS Computational Genomics Centre (ISRM) Division, Indian Council of Medical Research;

${ }^{5}$ Environmental Toxicology Laboratory, ICMR-National Institute of Pathology, New Delhi 110029;

${ }^{6}$ Department of Medical Elementology and Toxicology, Jamia Hamdard, New Delhi 110062;

${ }^{7}$ Department of Dermatology and STD, Vardhman Mahavir Medical College, Safdarjung Hospital, New Delhi 110029, India
\end{abstract}

Received May 20, 2019; Accepted July 31, 2020

DOI: $10.3892 /$ ijmm.2020.4771

\begin{abstract}
Psoriasis is a common chronic inflammatory skin disease affecting $>125$ million individuals worldwide. The therapeutic course for the disease is generally designed upon the severity of the disease. In the present study, the gene expression profile GSE78097, was retrieved from the National Centre of Biotechnology (NCBI)-Gene Expression Omnibus (GEO) database to explore the differentially expressed genes (DEGs) in mild and severe psoriasis using the Affy package in R software. The Kyoto Encyclopaedia of Genes and Genomes (KEGG) pathways of the DEGs were analysed using clusterProfiler, Bioconductor, version 3.8. In addition, the STRING database was used to develop DEG-encoded proteins and a protein-protein interaction network (PPI). Cytoscape software, version 3.7.1 was utilized to construct a protein interaction association network and analyse the interaction of the candidate DEGs encoding proteins in psoriasis. The top 2 hub genes in Cytohubba plugin parameters were validated using immunohistochemical analysis in psoriasis tissues. A total of 382 and 3,001 dysregulated mild and severe psoriasis DEGs were reported, respectively.
\end{abstract}

Correspondence to: Dr Arun Kumar Jain, Biomedical Informatics Centre, ICMR-National Institute of Pathology, New Delhi 110029, India

E-mail:drakjain@gmail.com

Professor George Thomas, Department of Molecular and Cellular Engineering, Sam Higginbottom University of Agriculture, Technology and Sciences, Prayagraj (Allahabad), Uttar Pradesh 211007, India E-mail: georgethomas@shiats.edu.in

Key words: psoriasis, psoriasis vulgaris, differentially expressed gene, hub genes, cytoscape
The dysregulated mild psoriasis genes were enriched in pathways involving cytokine-cytokine receptor interaction and rheumatoid arthritis, whereas cytokine-cytokine receptor interaction, cell cycle and cell adhesion molecules were the most enriched pathways in severe psoriasis group. PL1N1, TLR4, ADIPOQ, CXCL8, PDK4, CXCL1, CXCL5, LPL, AGT, LEP were hub genes in mild psoriasis, whereas BUB1, CCNB1, CCNA2, CDK1, CDH1, VEGFA, PLK1, CDC42, CCND1 and CXCL8 were reported hub genes in severe psoriasis. Among these, CDC42, for the first time (to the best of our knowledge), has been reported in the psoriasis transcriptome, with its involvement in the adaptive immune pathway. Furthermore, the immunoexpression of CDK1 and CDH1 proteins in psoriasis skin lesions were demonstrated using immunohistochemical analysis. On the whole, the findings of the present integrated bioinformatics and immunohistochemical study, may enhance our understanding of the molecular events occurring in psoriasis, and these candidate genes and pathways together may prove to be therapeutic targets for psoriasis vulgaris.

\section{Introduction}

Psoriasis is a common chronic inflammatory skin disease that affects $>125$ million individuals worldwide (1). It is a multifactorial disease (2), with both genetic and environmental factors contributing to its development. Beyond its dermatological manifestations, psoriasis has a negative impact on the quality of life of affected subjects and has been found to be an independent risk factor for cardiovascular diseases, psoriatic arthritis, atherosclerosis, diabetes and insulin resistance, hypertension, metabolic syndrome, myocardial infarction and obesity, which cause an immense disease burden on patients (3-6).

Chronic plaque psoriasis, referred as psoriasis vulgaris, is characterized by well-demarcated, erythematous, scaly plaques, which can involve any part of the skin, but most 
commonly the extensor surfaces (such as the elbows and knees) and scalp. Psoriasis is distinguished into mild versus moderate to severe, based on clinical evaluation methods, such as the extent of the affected skin surface. An affected body surface area $>10 \%$ of the body surface signifies moderate to severe disease with a Psoriasis Area and Severity Index (PASI) score of 12 being a minimum for entry into several clinical trials of systemic immune modulators (7). Psoriatic skin inflammation is considered to develop as a result of abnormal communications between infiltrating immune cells and activated keratinocytes. Th17 cells and their secreted cytokines, interleukin (IL)-17 and IL-22, in synergy with interferon (IFN) $-\gamma$ and tumour necrosis factor- $\alpha$ (TNF- $\alpha$ ), have been identified as central players in the disease pathogenesis $(8,9)$. Several researchers, including Bowcock et al (2001), Oestreicher et al (2001), Zhou et al (2003), Kulski et al (2005), Mee et al (2007), Yao et al (2008), Gudjonsson et al (2010) and Choudhary et al (2020) (10-17) have used microarray methods to explore psoriasis lesional transcriptome in order to elucidate the gene expression profile. In their studies, they elucidated the gene expression profiles of psoriasis lesions and highlighted pathways and differentially expressed genes (DEGs) that were mainly involved in cell adhesion and diapedesis, the role of IL-17A in psoriasis, atherosclerosis signalling and cytokine signalling in immune cells.

Additionally, Coda et al (2012) (18) examined the gene expression profiles in the blood of patients with mild-moderate psoriasis and found that the transcriptome of mild-moderate disease contains several genes implicated in previously reported transcriptome of lesional psoriasis.

Alterations in gene expression, although with a lower number of DEGs, have been detected even in non-lesional, healthy-looking skin of patients with psoriasis, suggesting a 'pre-psoriatic' state of non-lesional skin (19). The present study aimed to investigate the gene expression patterns between patients with moderate and severe psoriasis. The progression of the shared gene expression profiles among the mild and severe diseased state was also explored. With the combination of the traditional DEGs method and protein-protein interaction of microarray data from the Gene Expression Omnibus (GEO), the present study identified the hub genes among mild and severe psoriasis. The proteins of these identified hub genes were further evaluated for qualitative immunolocalization in psoriasis tissue biopsies. The present study also analysed various KEGG pathways involved in the disease. Taken together, these hub genes and pathways may represent potential biomarkers and drug targets.

\section{Materials and methods}

Gene expression profile retrieval and pre-processing. The gene expression profile dataset, GSE78097, was downloaded from GEO (http://www.ncbi.nlm.nih.gov/geo/). The retrieved expression profile is based on the data deposited from Rockefeller University, Laboratory for Investigative Dermatology, USA (20). The GSE record was generated on the GPL570 platform (Affymetrix Human Genome U133 plus 2.0 Array).

The raw data files used for the analysis was imported into $\mathrm{R}$, version 3.5.3. Background correction, normalization and $\log 2$ transformation were performed based on the robust multiarray average method embedded in the Affy package (21) in Bioconductor version 3.8 (https://bioconductor.org/). The Benjamini and Hochberg method was implemented in the limma package to calculate the false discovery rate (FDR) (22). Statistically significant DEGs were defined with an FDR $<0.05$ and $[\mathrm{FC}] \geq 2$ as the cut-off criterion. For any gene with multiple corresponding probes, the mean expression value of all probes was considered as its final expression value (23). Principal Component analysis (PCA) was also conducted on the DEGs to determine the orthogonal linear transformation of the input data such that patterns of variability can be investigated in the given dataset.

Gene ontology and pathway enrichment analysis. The DEGs were analysed in clusterProfiler to assign gene ontology terms for their possible role in biological process, molecular function and cellular components (24). The Kyoto Encyclopaedia for Genes and Genomes (KEGG)-based screening was also performed to identify the role of the DEGs in various metabolic pathways (25).

Genes-disease network of DEGs. The DEGs were annotated based on gene-disease association data from knowledge bases viz. genome wide association study catalogue (GWAS) (26), genetics association database (GAD) (27), comparative toxico-genomics database (28), ClinVar (29) and UniProt (30) through DisGeNET (31) and target validation (32). The gene-disease network was inspected by integrating the data into Cytoscape v3.7.1.

Integration of the protein-protein interaction (PPI) network, modular analysis and identification of significant candidate genes and pathways in psoriasis. The STRING database (available online: http://string-db.org) (33) was employed to develop DEG-encoded proteins and the PPI network. Cytoscape software, version 3.7.1 (34) was utilized to construct the protein interaction association network and analyze the interaction association of the candidate DEGs encoding proteins in psoriasis. The CytoHubba plugin in Cytoscape was employed to identify hub genes. The hub scores for the PPI network were calculated by using the topology of 12 built-in centrality parameters i.e., MCC, DMNC, MNC, Degree, EPC, Bottleneck, Eccentricity, Closeness, Radiality, Betweenness, Stress, and Clustering Coefficient as previously described by Yang et al (2018) (35). The top 20 genes in each 12 built-in centrality parameters were sorted and genes identified in at least 6 centrality indexes were considered as high-confidence hub targets with possible biological significance (36). Molecular complex detection (MCODE) was used to screen the modules of the PPI network with a degree cut-off of 10 , a node score cut-off of 0.2 , a k-core of 2 and a maximum depth of 100. The Gene Ontology (GO) analysis of top 3 modules was performed by STRING Enrichment plugin. FDR $<0.05$ was set as the cut-off criteria for GO analysis.

Reagents. Primary antibodies against CDK1 (orb213697) and CDH1 (orb213705) were procured from Biorbyt and the Super Sensitive ${ }^{\mathrm{TM}}$ Polymer-IHC detection kit was obtained from Biogenex. Xylene, ethyl alcohol, methanol and $\mathrm{H}_{2} \mathrm{O}_{2}$ were procured from Merck KGaA. 

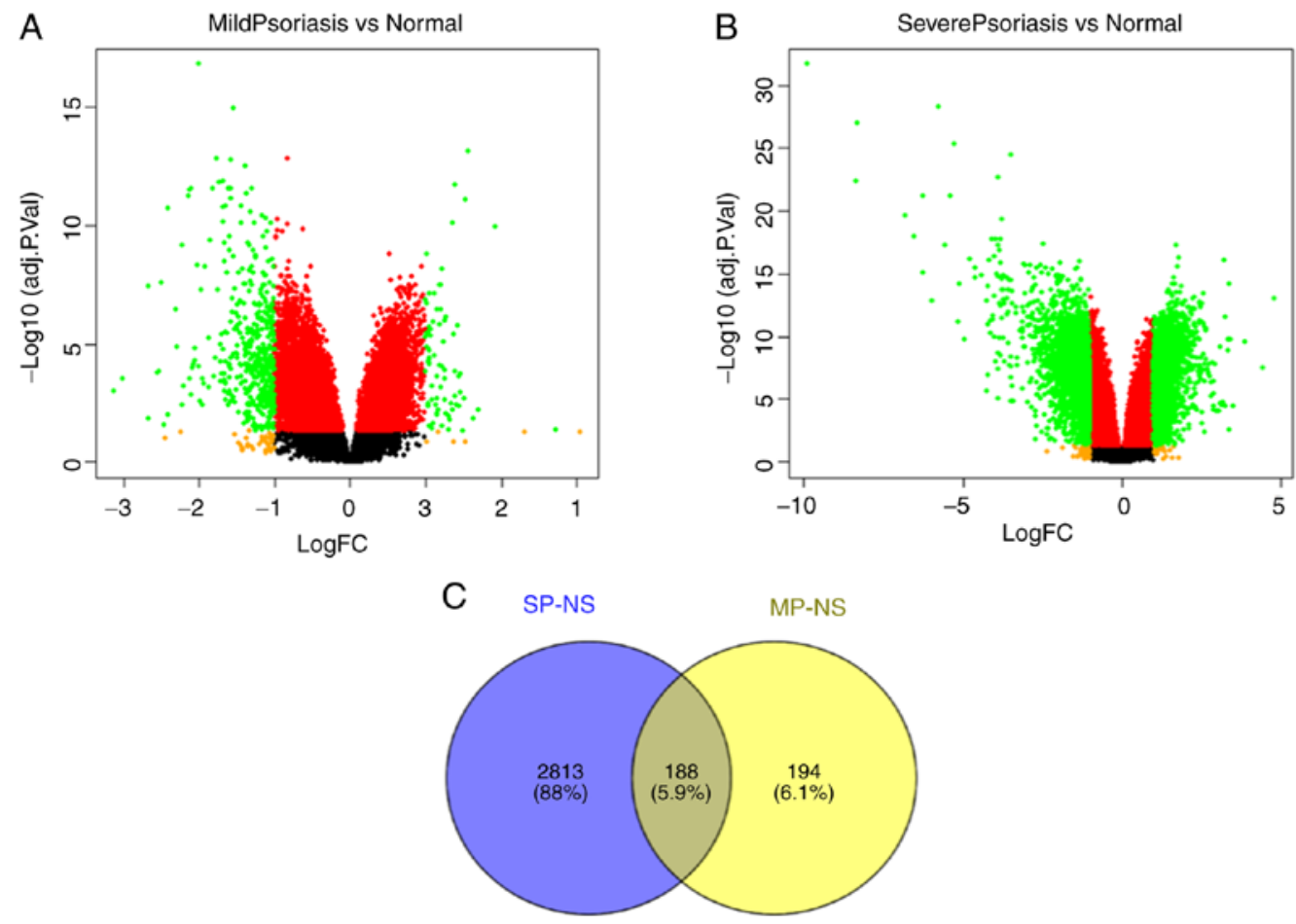

Figure 1. (A) Volcano plot showing DEGs between mild psoriasis and normal samples, (B) volcano plot showing DEGs between severe psoriasis and normal samples, (C) Venn diagram representing DEGs in both severe (blue colour) and mild (yellow colour) group. The intersection part represents the shared DEGS between both the groups. DEGs, differentially expressed genes.

Patient information. A total of 8 patients with a clinical diagnosis of psoriasis vulgaris [4 males (50\%) and 4 females $(50 \%)$ ] were included in the present study. The tissue biopsy was collected from the Out-Patient Department of Dermatology, Safdarjung Hospital, New Delhi. Their ages ranged between 28-49 years and the tissue biopsy sample collection was completed within 40 days (January, 2020 to February, 10, 2020). Study subjects with any habits of smoking, drug addiction and alcohol abuse were eliminated. Subjects with any other types of chronic inflammation, HIV, and the presence of haematological, hepatic or renal disorders were also excluded. Additionally, any patient with clinical evidence of infection during a week prior to sample collection and or undergoing any systematic or biological therapy were also not enrolled in the present study. Informed written consent was obtained from the patients and tissue biopsy samples were obtained from lesioned skin margins; i.e., psoriasis involved and uninvolved. Ethical approval from the Ethics Committee of Vardhman Mahavir Medical College and Safdarjung Hospital, New Delhi (IEC/VMMC/SIH/Project/2018/1094) was obtained.

Immunohistochemistry. Formalin-fixed and paraffin-embedded 5 - $\mu \mathrm{m}$-thick tissue slides were prepared. The sections were deparaffinized in xylene and hydrated in ethyl alcohol of decreasing concentrations. Endogenous peroxidase activity was quenched in $3 \% \mathrm{H}_{2} \mathrm{O}_{2}$ in methanol for $30 \mathrm{~min}$ at room temperature. For antigen retrieval, the sections were heated in citrate buffer $(\mathrm{pH} 6.0)$ at $100^{\circ} \mathrm{C}$ for $20 \mathrm{~min}$. The sections were then washed in PBS ( $\mathrm{pH}$ 7.4) thrice for 5 min each and blocked with Power Block for $30 \mathrm{~min}$. The sections were then incubated with primary polyclonal antibodies (pAbs), CDK1 and CDH1 (dilution of 1:50 in PBS for both the pAbs). One slide of serial sections from each case was run simultaneously as a negative control in which the primary pAb was omitted. Subsequently, the sections were incubated with Super Enhancer and Polymer-HRP for $30 \mathrm{~min}$ at $37^{\circ} \mathrm{C}$. DAB chromogen was applied on the antigen-antibody complex for 5-10 min and was immediately terminated when brown colouration was developed. The slide was further counterstained with Mayer's haematoxylin and was again dehydrated in ethyl alcohol of increasing concentration and cleared with xylene. Finally, slides were air dried, further mounted by DPX medium and examined under light microscope (Olympus, BX-43). Images were captured using a digital camera (Olympus, E-520).

\section{Results}

Data pre-processing and identification of DEGs. GSE78097 was retrieved from NCBI-GEO. The microarray data of GSE78097 included 14 mild plaque psoriasis lesional skin biopsy tissues, 13 severe plaque psoriasis lesional skin biopsy tissues and 6 normal skin tissues from a North-American population. Using an FDR $<0.05$ and $[\log \mathrm{FC}] \geq 2$ as the cut-off criterion, 382 differentially expressed mild psoriasis genes were obtained of which 49 were upregulated and 333 were downregulated whereas 3,001 dysregulated gene were extracted in severe psoriasis of which 1,184 were upregulated and 1,817 were downregulated (Fig. 1A and B). In total, 188 differentially expressed genes were shared between the severe and mild psoriasis group (Fig. 1C). Among these, the expression levels of 73 genes were downregulated in mild psoriasis and with an increase in the degree of disease severity, their expression increased abruptly. The majority of these DEGs were reportedly involved in the signal transduction pathway, cytokine signalling and in pathways of 


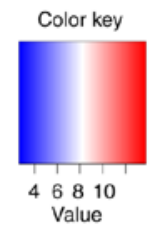

A

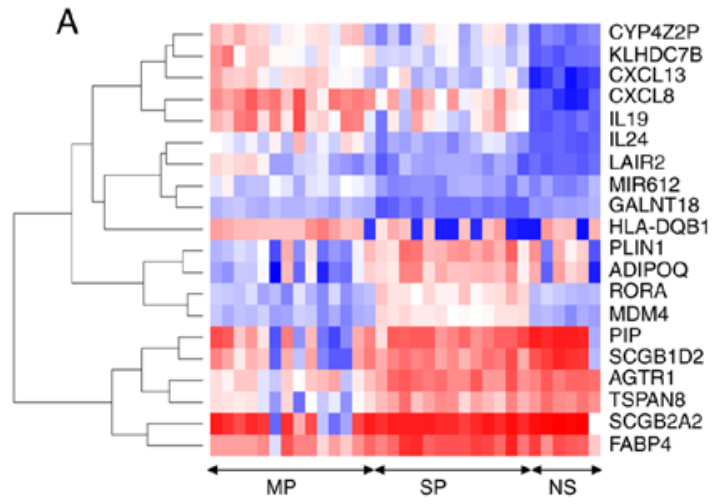

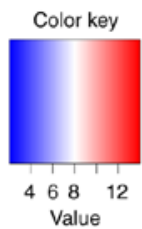

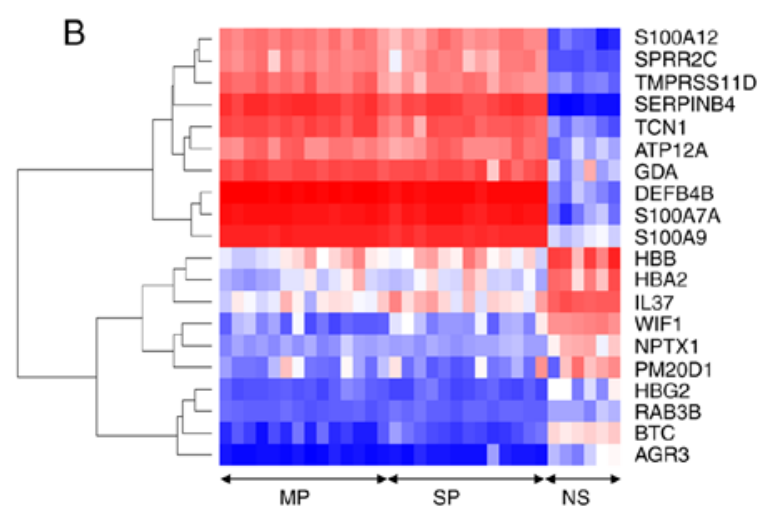

Figure 2. Heatmap of top 20 dysregulated (10 upregulated and 10 downregulated) DEGs represents expression of (A) mild psoriasis DEGs (B) severe psoriasis DEGs. Red colour signifies upregulation and blue signifies the downregulation of DEGs. DEGs, differentially expressed genes.

innate and adaptive immune system. The expression profiles of top 20 dysregulated gene from both groups in each sample was visualized as heatmap (Fig. 2). PCA analysis of these genes presented a visually apparent association between the 2 groups (Fig. 3) and these dysregulated genes can be used to classify the sample on diseased state depending upon its expression profile.

DEG GO analysis in severe and mild psoriasis. DEG GO analysis was performed using the Bioconductor package, clusterProfiler, which aptly analysed and visualized its functional profiles (GO and KEGG). The dysregulated DEGs for both the groups were classified into 3 categories explaining molecular function, biological process and cellular components. The biological process among dysregulated mild psoriasis DEGs are pre-dominantly enriched in response to peptide, peptide hormone, muscle system process, ossification and the regulation of lipid metabolic process, whereas in severe psoriasis they are found to be involved in T-cell activation and had positive implication in organelle fission and chromosome segregation. In the molecular functional group, the dysregulated DEGs among mild psoriasis group was reportedly involved in receptor regulator activity, receptor ligand activity, cytokine and chemokine activity. Notably, the severe psoriasis genes were enriched in cell adhesion molecule binding, actin binding, cytokine as well in chemokine activity. Among the cellular component group, mild psoriasis genes were only enriched in proteinaceous extracellular matrix, however, the dysregulated gene among severe psoriasis group was highly enriched in cell-cell junction, spindle and condensed chromosome. The GO annotation of both the groups is illustrated in Fig. 4.

Signalling pathway enrichment analysis. The dysregulated mild psoriasis genes were mainly found to be enriched in pathways involving cytokine-cytokine receptor interaction, rheumatoid arthritis, adrenergic signalling in cardiomyocytes whereas cytokine-cytokine receptor interaction, cell cycle, NOD-like receptor signalling pathway and cell adhesion
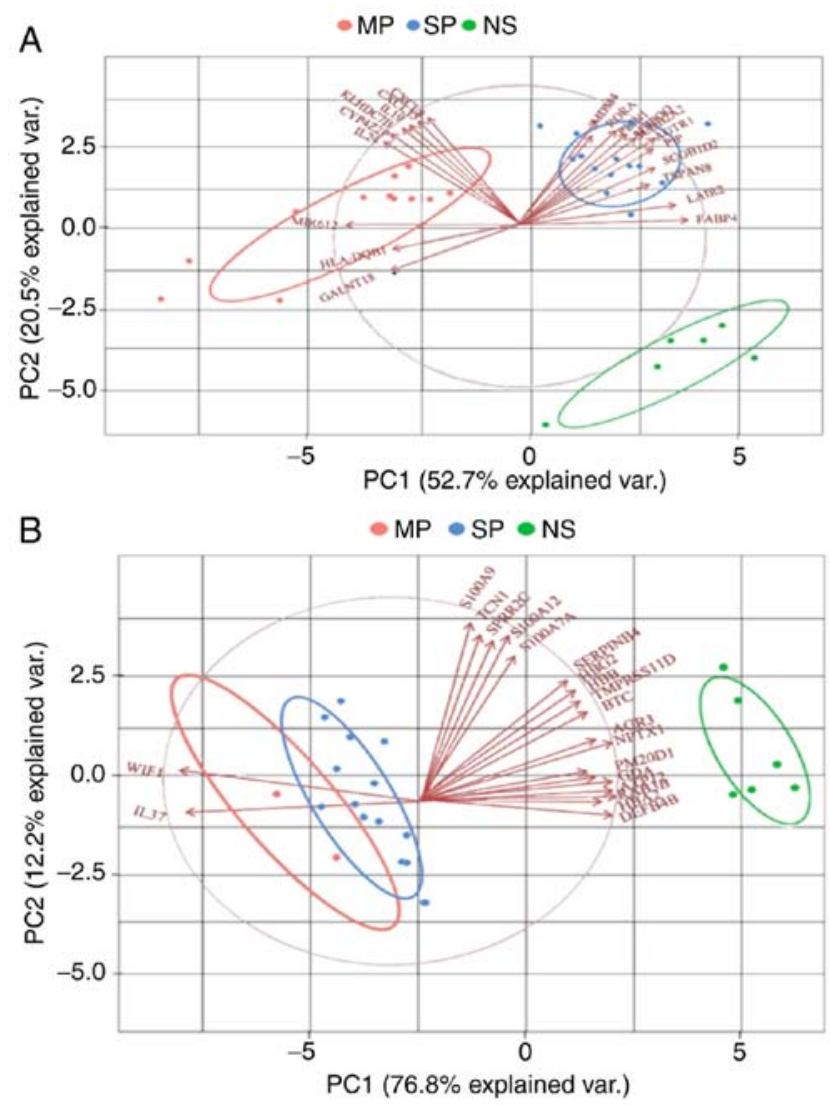

Figure 3. The principal component plot of top 20 dysregulated genes. (A) Mild psoriasis, (B) severe psoriasis of 33 samples distinguishes the DEGS between the sample sub-types. DEGs, differentially expressed genes.

molecules were comparatively found to be the most enriched pathways among DEGS in the severe psoriasis group (Fig. 5).

Gene-disease network of DEGs. The identified DEGs among both groups were mapped to the validated disease genes in DisGeNET and target validation human genetic disorder 


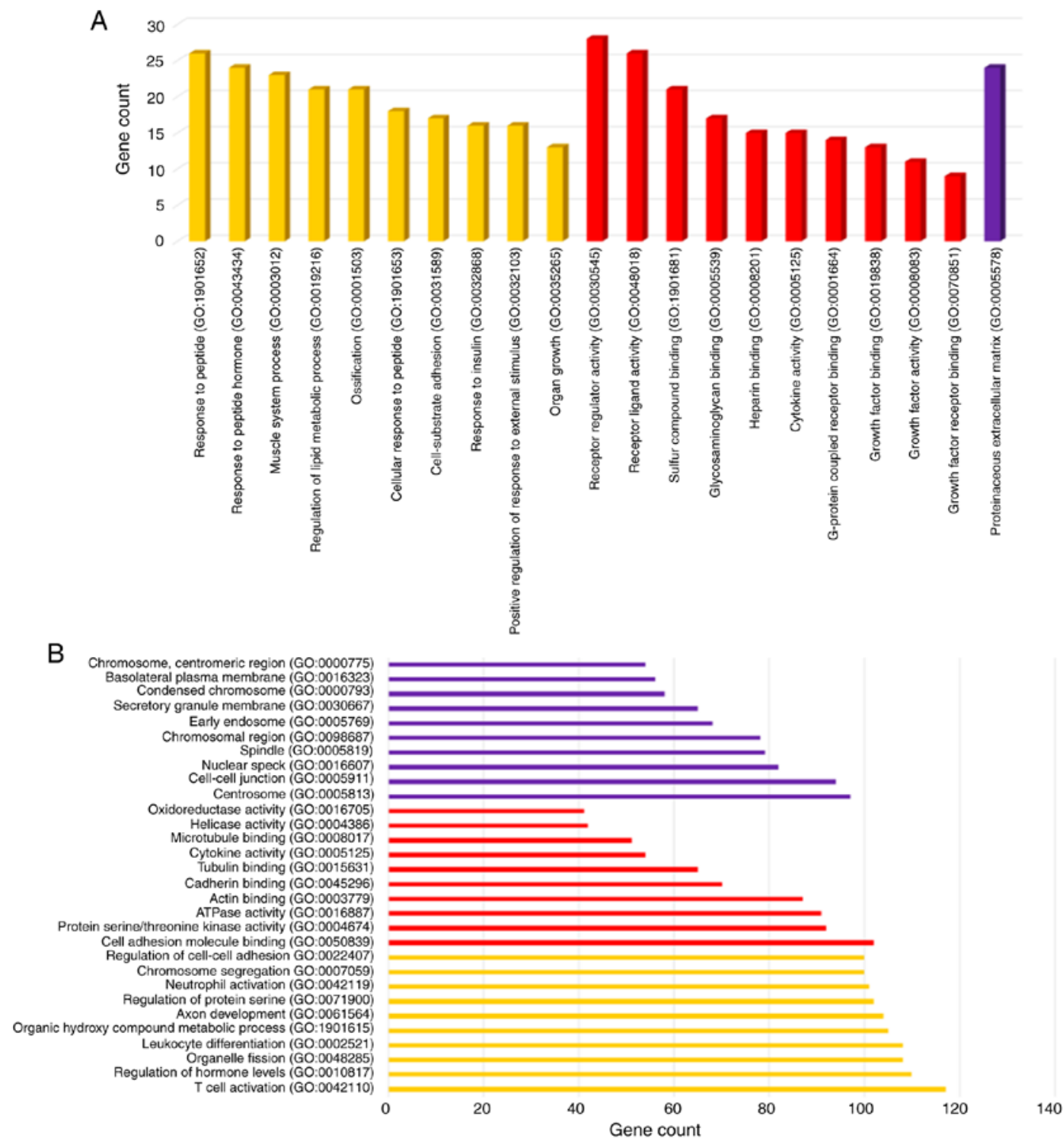

Figure 4. Gene ontology (GO) analysis and significant GO terms of DEGs in mild and severe psoriasis. (A) GO analysis classified the mild psoriasis DEGs, (B) GO analysis classified the severe psoriasis DEGs into 3 groups (i.e., biological process, molecular function, and cellular component). Yellow bars represent biological process, red bars molecular function and purple bars cellular component. DEGs, differentially expressed genes.
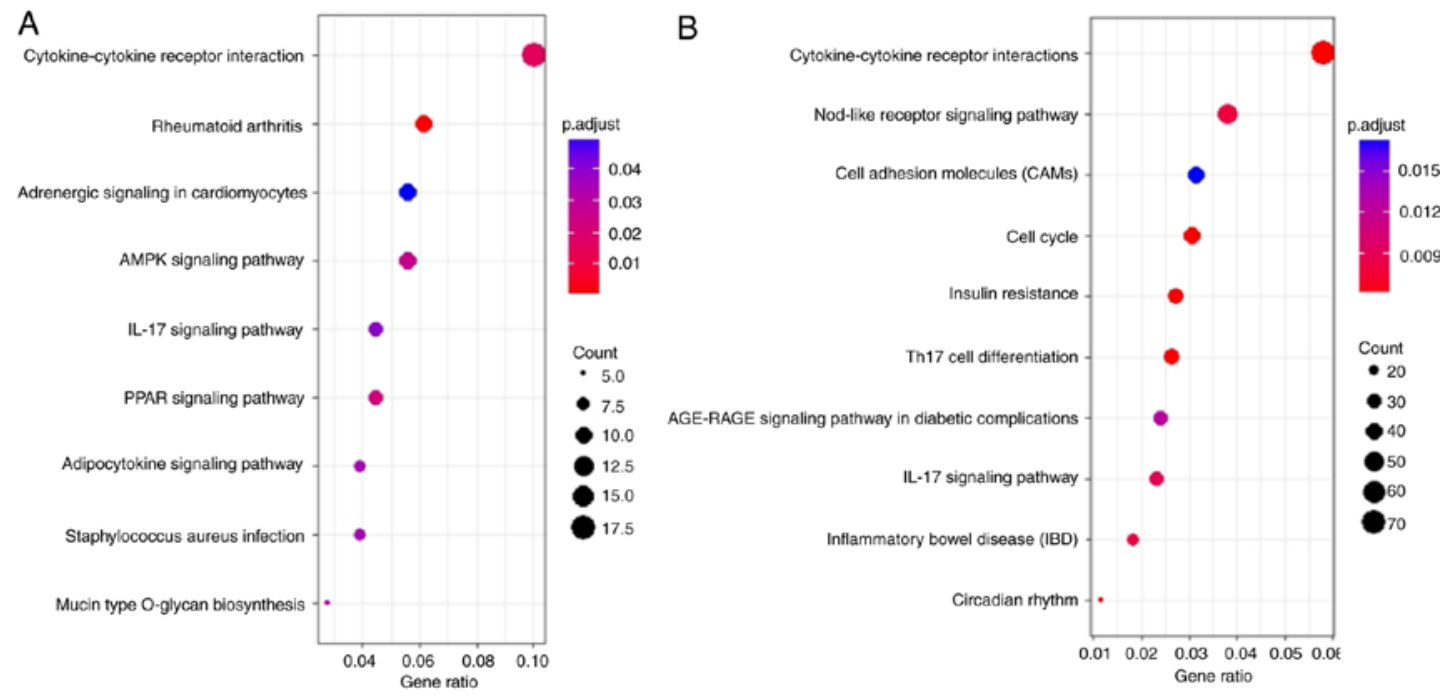

Figure 5. Significantly enriched pathway terms of DEGs. (A) Mild psoriasis, and (B) severe psoriasis. DEGs functional and signalling pathway enrichment was conducted using online website of KEGG Pathway in clusterProfiler. DEGs, differentially expressed genes. 


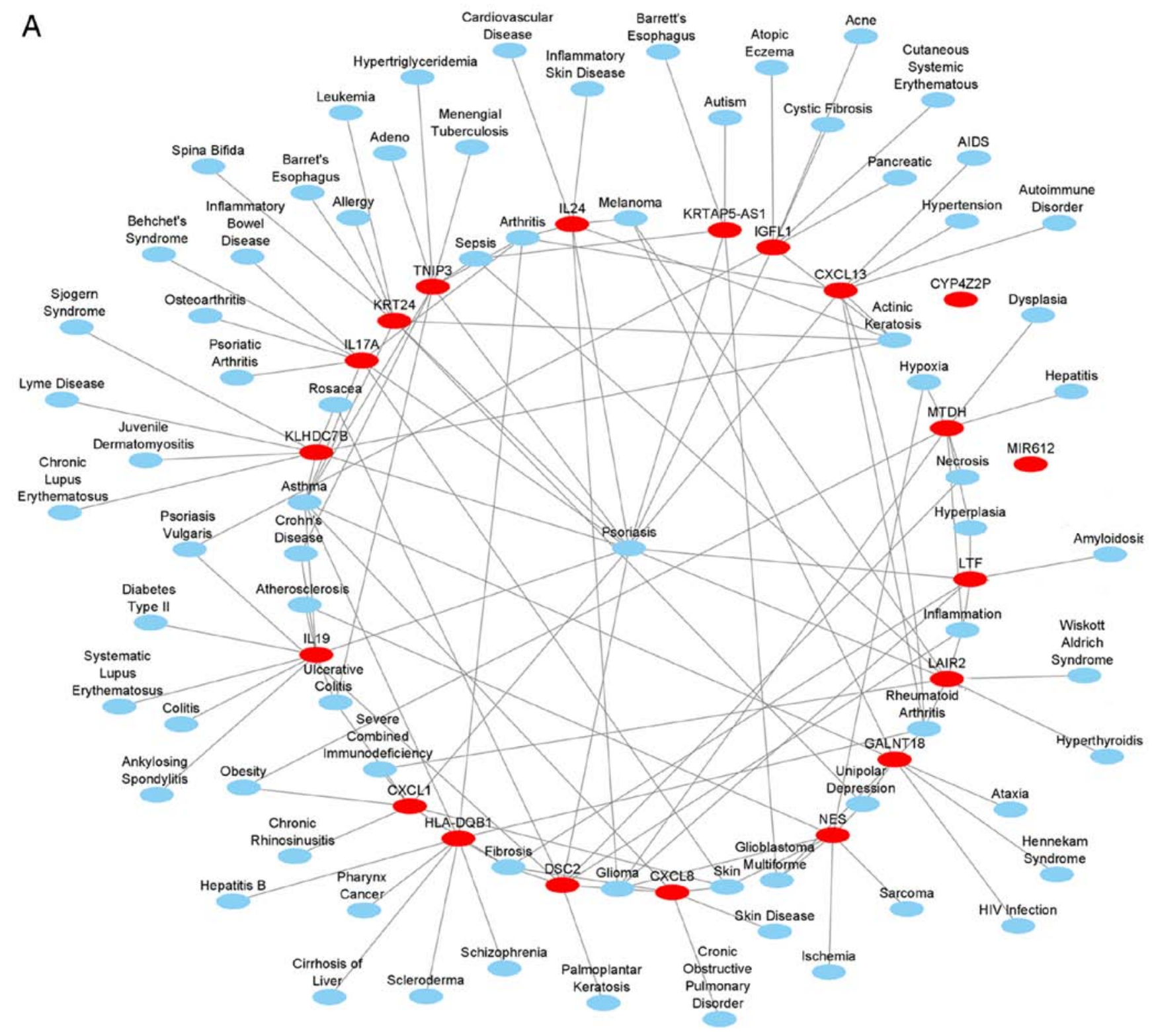

Figure 6. Continued.

databases. This analysis confirmed that 301 mild psoriasis $(78.79 \%)$ and 1,700 severe psoriasis $(56.65 \%)$ validated genes present in these 2 databases had also been identified as psoriasis-associated DEGs in the expression analysis in the present study. This indicates that the identified DEGs were appropriate to signify the disease sub-types. The gene-disease network for 20 highest ranking genes associated to diseased state based on the fold change value were reported to be associated with another dermal disease rosacea and several auto-immune disorders including Crohn's disease, rheumatoid arthritis and Wiskott-Aldrich syndrome (Fig. 6).

Key candidate genes and pathway identification with DEG PPI and module analysis. In the mild psoriasis group, 382 DEGs (49 upregulated and 333 downregulated) with 347 nodes and 608 edges and 3,001 DEGs (1,184 upregulated and 1,817 downregulated genes) in the severe psoriasis group containing 2,644 nodes and 34,473 edges were filtered from the PPI network complex. The nodes indicate the genes, and the edges indicate the interactions between the genes. EGFR is the highest connected node having 273 degree among severe psoriasis category while LEP is most connected node in mild psoriasis group. The constructed network was analysed using the Cytohubba plugin and the DEGs present in $\geq 6$ parameters were considered as hub genes. The top 10 hub genes for mild psoriasis and severe psoriasis are illustrated as Fig. 7. Module analysis on the PPI network revealed 6 clusters in mild psoriasis and 44 clusters in severe psoriasis.

The top 3 modules of each category were further examined. Pathway enrichment analysis of the modules revealed that each module is functionally related. The modules of mild psoriasis are related to signalling pathways, inflammatory response, immune system whereas the module of severe psoriasis group was mainly related to cell cycle regulation, post translational modification, cytokine-mediated signalling pathway, cell surface receptor signalling pathway and $G$ protein-coupled receptor signalling pathway (Table I). 


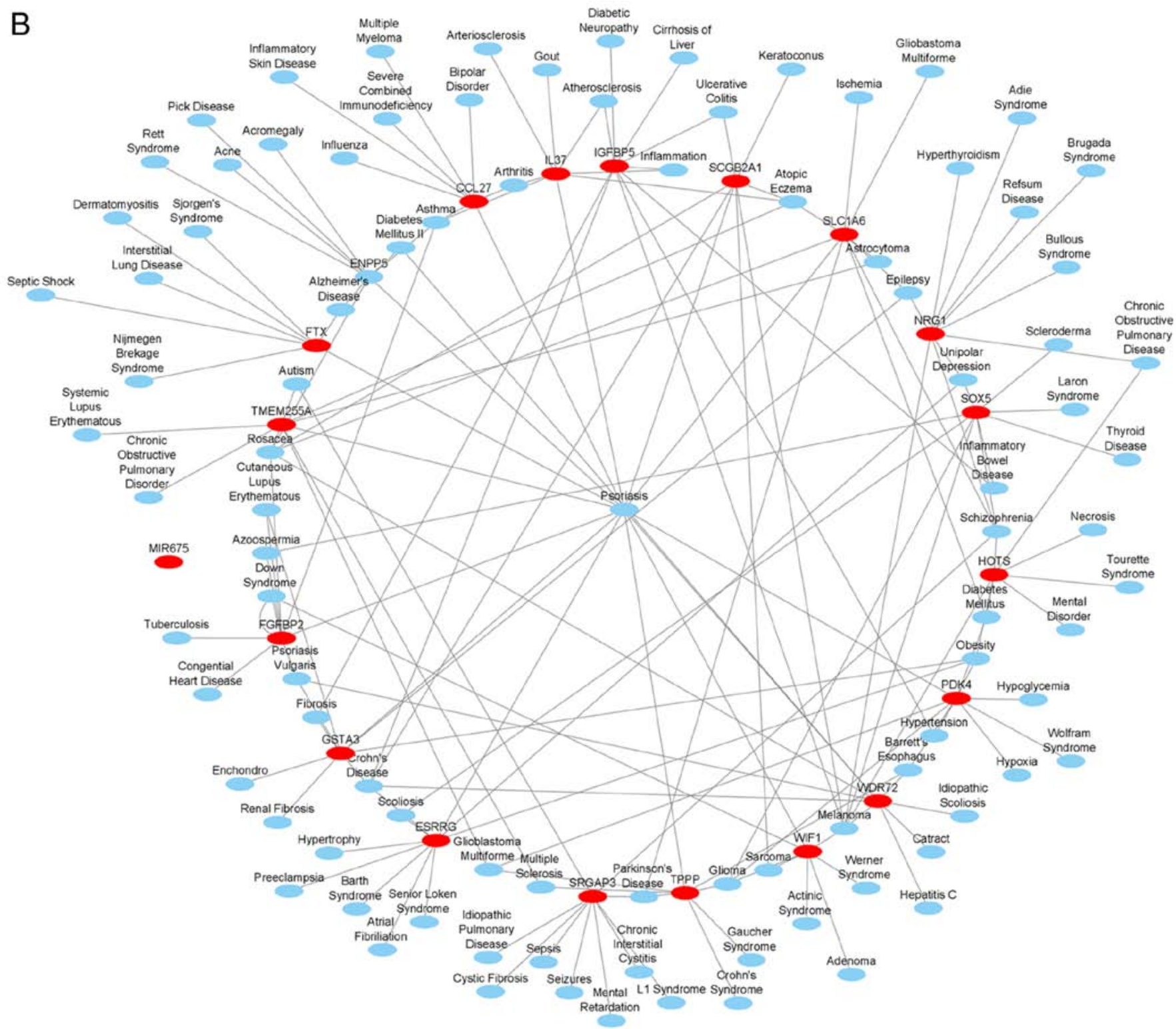

Figure 6. Gene disease network of the differentially expressed genes. (A) Mild-psoriasis vulgaris sub type. (B) Severe-psoriasis vulgaris sub type.

Immunohistochemical expression of CDK1 and CDH1. CDK1 expression was clearly observed in the basal layers of the epidermis showing positive nuclear staining. CDK1 expression visibly revealed positive nuclear staining in the basal layer of the epidermis. CDK1 immunoreactivity was evident at regular intervals in the non-lesional epidermis however intensely strong positive nuclear staining was visible among several stained nuclei in the basal layer of psoriatic skin lesions (Fig. 8). CDH1 immunoreactivity was observed at different layers of the epidermis and the immunostaining was localized in the nuclear, as well as the cytoplasmic region. A spatial decrease in CDH1 expression was reported in the epidermis of lesional psoriasis compared with non-lesional epidermis, specifically in the basal cell and upper granular layers of epidermis (Fig. 9). Therefore, as per the immunohistochemical analysis, a marked decrease in $\mathrm{CDH} 1$ expression was noted in the epidermis of psoriasis skin lesions. The distribution and expression patterns of both the antibodies, CDK1 and CDH1, were summarized as the overexpression of CDK1 in psoriasis skin lesions, while there was a marked decrease in CDH1 expression in lesional psoriatic skin samples as compared to non-lesional samples. The results of the immunohistochemical analysis were in agreement with the transcriptome analysis, where CDK1 was reportedly upregulated and CDH1 was downregulated severe psoriasis.

\section{Discussion}

In the present study, gene expression in the GSE78097 dataset was analysed and gene expression between psoriasis lesions was compared based upon the severity of the disease, i.e., mild and severe psoriasis vulgaris. The present study identified 382 and 3,001 differentially expressed dysregulated genes in the mild and severe psoriasis vulgaris group, respectively with 188 overlapping DEGs.

HLA-DQB1 was the most upregulated and ADIPOQ the most downregulated gene among the mild psoriasis group, whereas WIF1 was the most upregulated and SERPINB4 the 


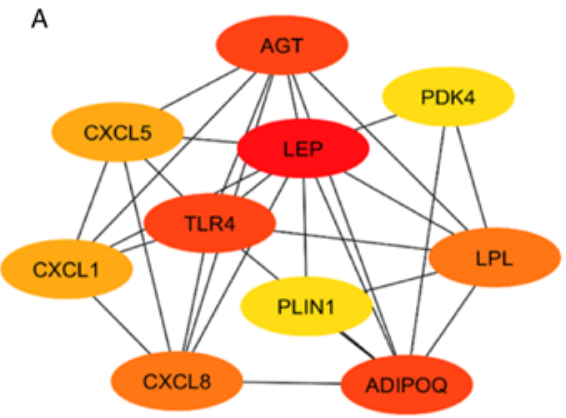

\begin{tabular}{|c|c|}
\hline GENE NAME & DEGREE \\
\hline LEP & 28 \\
\hline CXCL8 & 27 \\
\hline ADIPOQ & 21 \\
\hline AGT & 20 \\
\hline LPL & 20 \\
\hline TLR4 & 17 \\
\hline CXCL1 & 15 \\
\hline PLIN1 & 11 \\
\hline CXCL5 & 10 \\
\hline PDK4 & 10 \\
\hline
\end{tabular}

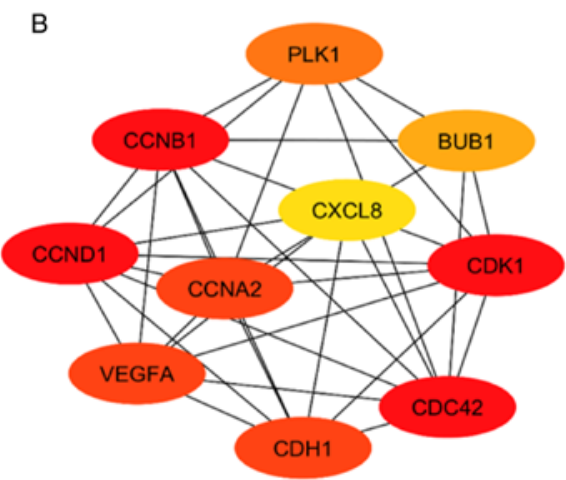

\begin{tabular}{|c|c|}
\hline GENE NAME & DEGREE \\
\hline CDK1 & 250 \\
\hline VEGFA & 231 \\
\hline CCNB1 & 221 \\
\hline CDC42 & 211 \\
\hline PLK1 & 207 \\
\hline CCNA2 & 196 \\
\hline CXCL8 & 190 \\
\hline CDH1 & 186 \\
\hline CCND1 & 175 \\
\hline BUB1 & 168 \\
\hline
\end{tabular}

Figure 7. Top 10 hub genes with DEGs degree from the PPI network. (A) Mild psoriasis. (B) Severe psoriasis.
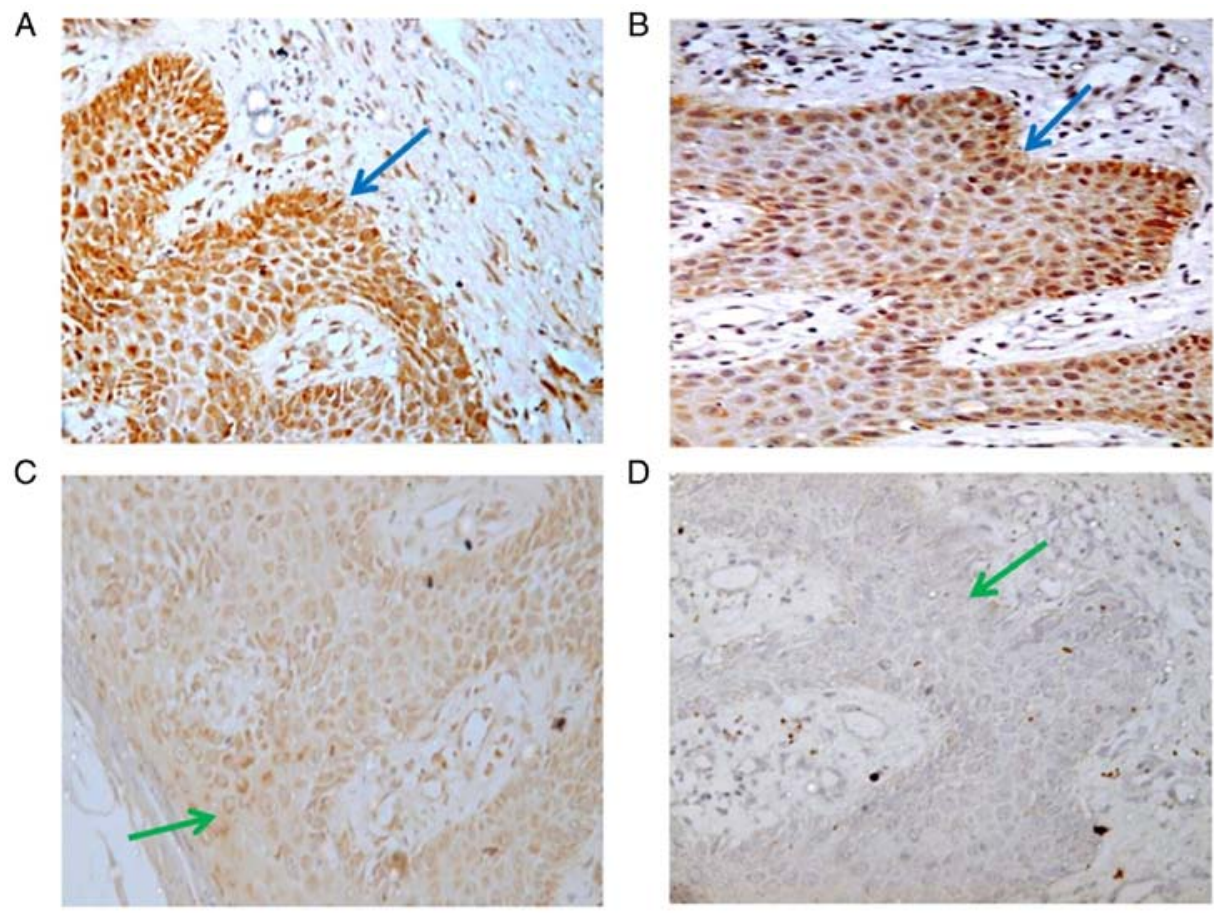

Figure 8. CDK1 immunoreactivity in psoriatic skin lesions and non-lesional samples. CDK1 immunoexpression and localization (blue and green arrows) in (A and B) psoriatic skin lesions and (C and D) non-lesional or normal skin tissue; magnification $\mathrm{x} 40$.

most downregulated gene among severe psoriasis group. The analysis of the top dysregulated genes in both mild psoriasis and severe psoriasis revealed that $\mathrm{T}_{\mathrm{H}} 17$-regulated cytokine (IL19 and CXCL13) and $\mathrm{T}_{\mathrm{H}} 1$-regulated cytokines (IL24 and CXCL8) were highly expressed at the time of disease initiation i.e., in mild psoriasis. The higher expression of driver inflammatory cytokines and negative immune regulators can be attributed to increased $\mathrm{T}$-cell proliferation and the creation of more skin-homing memory T-cells. These T-cell expansions may be enabled by having less effective immune regulation in patients that develop more extensive psoriasis lesions. The finding of the present study are well supported by those of Kim et al (2016) (20) and Lowes et al (2014) (37), who in their studies, identified the presence of these cytokines in 
Table I. Enriched KEGG pathway in top 3 clusters of mild psoriasis and severe psoriasis.

A, Top 3 clusters in mild psoriasis

\begin{tabular}{|c|c|c|c|c|}
\hline Cluster & Description & $\begin{array}{l}\text { No. of genes } \\
\text { enriched }\end{array}$ & Enriched gene & FDR value \\
\hline \multirow[t]{5}{*}{ Cluster 1} & hsa05323: Rheumatoid arthritis & 5 & CXCL5, CXCL8, IL17A, TLR4, CXCL1 & 4.36E-10 \\
\hline & hsa04657: IL-17 signalling pathway & 4 & CXCL5, CXCL8, IL17A, CXCL1 & $1.70 \mathrm{E}-07$ \\
\hline & $\begin{array}{l}\text { hsa04060: Cytokine-cytokine } \\
\text { receptor interaction }\end{array}$ & 4 & CXCL5, CXCL8, IL17A, CXCL1 & $6.52 \mathrm{E}-06$ \\
\hline & hsa05134: Legionellosis & 3 & CXCL8, TLR4, CXCL1 & $6.52 \mathrm{E}-06$ \\
\hline & hsa05133: Pertussis & 3 & CXCL5, CXCL8, TLR4 & $9.75 \mathrm{E}-06$ \\
\hline \multirow[t]{4}{*}{ Cluster 2} & hsa03320: PPAR signalling pathway & 4 & FABP4, LPL, PCK1, ADIPOQ & $1.82 \mathrm{E}-07$ \\
\hline & $\begin{array}{l}\text { hsa04920: Adipocytokine signalling } \\
\text { pathway }\end{array}$ & 3 & PCK1, LEPR, ADIPOQ & $2.07 \mathrm{E}-05$ \\
\hline & hsa04152: AMPK signalling pathway & 3 & PCK1, LEPR, ADIPOQ & $6.93 \mathrm{E}-05$ \\
\hline & $\begin{array}{l}\text { hsa04932: Non-alcoholic fatty liver } \\
\text { disease (NAFLD) }\end{array}$ & 2 & LEPR, ADIPOQ & 0.0076 \\
\hline \multirow[t]{2}{*}{ Cluster 3} & hsa04658: Th1 and Th2 cell differentiation & 2 & PRKCQ, HLA-DPA1 & 0.004 \\
\hline & hsa04659: Th17 cell differentiation & 2 & PRKCQ, HLA-DPA1 & 0.004 \\
\hline
\end{tabular}

$\mathrm{B}$, Top 3 clusters in severe psoriasis

\begin{tabular}{|c|c|c|c|}
\hline Cluster & Description & $\begin{array}{c}\text { No. of genes } \\
\text { enriched }\end{array}$ & Enriched gene \\
\hline
\end{tabular}

Cluster 1 hsa04110: Cell cycle

hsa04114: Oocyte meiosis

hsa04914: Progesterone-mediated

oocyte maturation

hsa04115: p53 signalling pathway

hsa04218: Cellular senescence

Cluster 2 hsa04120: Ubiquitin-mediated proteolysis

Cluster 3 hsa04062: Chemokine signalling pathway

hsa04060: Cytokine-cytokine receptor interaction

hsa05164: Influenza A
20

CDC6, CCNB1, ESPL1, MCM4, MCM6, CCNA2, BUB1B, CCNB2, MAD2L1, PLK1, BUB1, CDC25A, CDC25C, TTK, ORC1, CDC20, PTTG1, CDK1, CHEK1, CDC45

12 AURKA, FBXO5, CCNB1, ESPL1, CCNB2, MAD2L1, PLK1, BUB1, CDC25C, CDC20, PTTG1, CDK1

10 AURKA, CCNB1, CCNA2, CCNB2, MAD2L1, PLK1, BUB1, CDC25A, CDC25C, CDK1

6

CCNB1, CCNB2, RRM2, CDK1, CHEK1, GTSE1

7

17 VHL, ANAPC5, UBE2S, FBXW11,

CCNB1, CCNA2, CCNB2, CDC25A, FOXM1, CDK1, CHEK1 UBE3C, UBA6, UBE2N, SOCS3, UBE2G2, UBA1, UBE2H, SIAH1, SMURF1, PARK2, KLHL13, UBE2F, ITCH

$20 \mathrm{CCR} 7, \mathrm{CCL} 27, \mathrm{ADCY} 8, \mathrm{CXCL} 13$, CCR5, CXCL3, CXCL10, CXCL11, STAT2, CXCR2, ADCY2, CCL20, STAT1, CXCL9, ADCY7, CXCL1, CXCR4, CXCR6, CXCL2, CCL4 CCR7, CCL27, CXCL13, CCR5, CXCL3, CXCL10, CXCL11, CXCR2, CCL20, CXCL9, CXCL1, CXCR4, CXCR6, CXCL2, CCL4

12 OAS3, IFIH1, CXCL10, STAT2, OAS2, STAT1, DDX58, RSAD2, IRF9, IRF7, MX1, OAS1
$5.25 \mathrm{E}-21$

4.35E-09

3.31E-05

2.70E-04

$1.11 \mathrm{E}-20$

1.37E-23

$1.64 \mathrm{E}-13$

7.10E-12 
Table I. Continued.

B, Top 3 clusters in severe psoriasis

\begin{tabular}{llcl}
\hline Cluster & \multicolumn{1}{c}{ Description } & $\begin{array}{c}\text { No. of genes } \\
\text { enriched }\end{array}$ & Enriched gene \\
\hline $\begin{array}{l}\text { hsa04621: NOD-like receptor signalling } \\
\text { pathway } \\
\text { hsa05160: Hepatitis C }\end{array}$ & $11 \quad$ & $\begin{array}{l}\text { OAS3, CXCL3, STAT2, OAS2, STAT1, } \\
\text { GBP1, CXCL1, IRF9, IRF7, OAS1, CXCL2 } \\
\text { OAS3, IRF1, STAT2, OAS2, STAT1, IFIT1, 2.79E-10 } \\
\text { DDX58, IRF9, IRF7, OAS1 }\end{array}$ \\
\hline
\end{tabular}
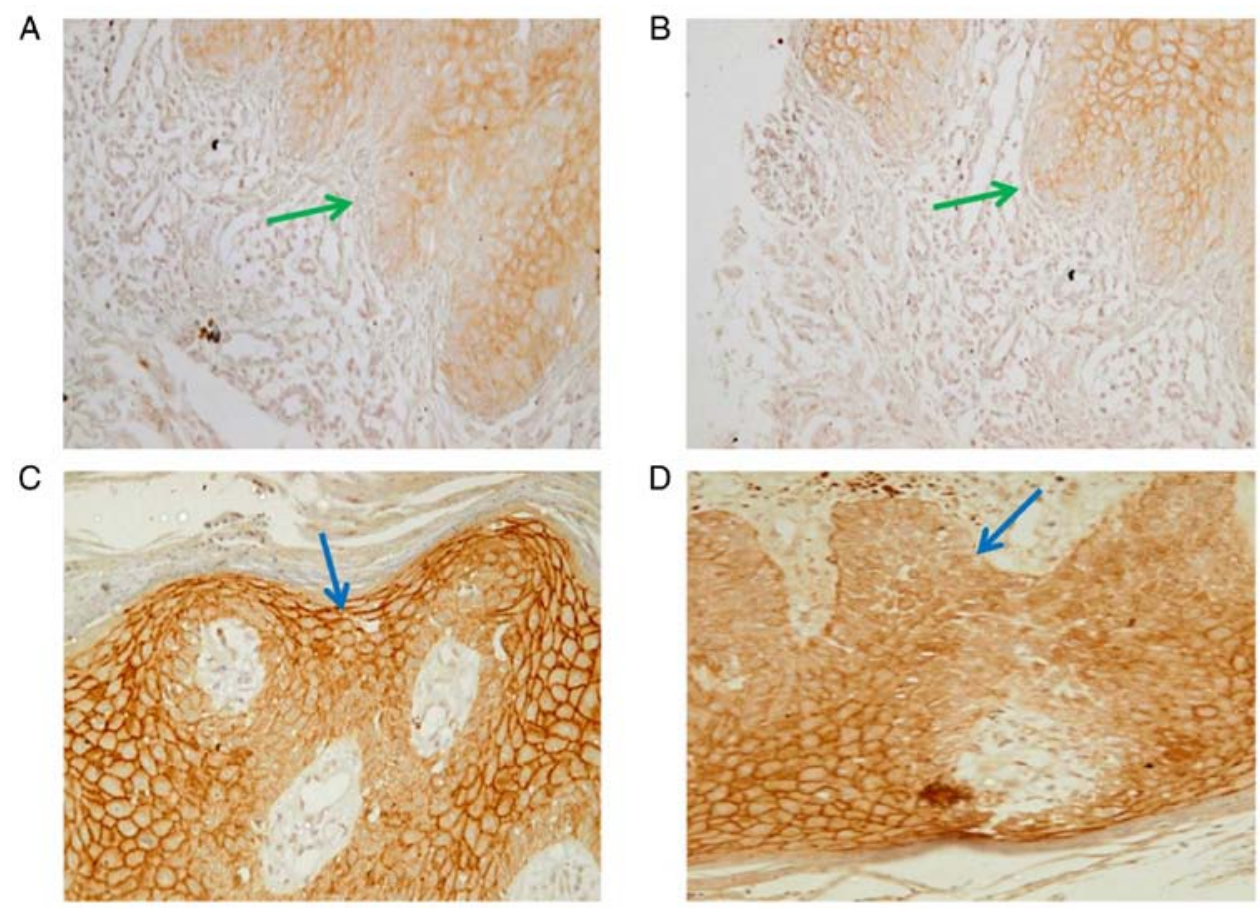

Figure 9. CDH1 immunoreactivity in human psoriatic skin tissue. CDH1 immunoexpression and localization (blue and green arrows) in (A and B) psoriatic skin lesions and (C and D) non-lesional or normal skin tissue; magnification $\mathrm{x} 40$.

disease initiation and with disease progression the expression of these cytokines decreased significantly. Moreover, among the 188 overlapping DEGs, 73 DEGs were reportedly downregulated in mild psoriasis and exhibited an upregulated fold change expression in the severe state. The majority of these genes (MIR675, PDK4, SLC1A6, WIF1, WDR72, CCL27 and ERBB4) are involved in signal transduction pathways, cytokine signalling and in pathways of innate and adaptive immune system (38-40).

The pathway enrichment analysis of DEGs involved with mild and severe psoriasis sub-types revealed several known pathways (Fig. 5A and B). The maximum enriched DEGs in both sub-types have been found in well-established cytokine-cytokine receptor interaction in psoriasis [Ogawa et al (2018), Chen et al (2015) and Tan et al (2017) (41-43)]. In the present study, the observation of the enriched pathways in mild psoriasis revealed pathways involved in Staphylococcus aureus infection. The pathway involved in Staphylococcus aureus approves a previously demonstrated (44), establishing the importance of anti-microbial peptides in the innate immunity of human skin. The findings of the present study were in line with those of a previous study by the authors (2), who established Streptococcus, another Gram-positive bacterium to be involved in the initiation of the disease through respiratory tract. In addition, the observation made for the top dysregulated DEGs in the present study was well supported by network analysis. PPI analysis identified EGFR as most connected gene with highest node degree 273 in the severe psoriasis group. Johnston et al (2011) (45) also reported that members of EGF to be overexpressed in wounded skin and several authors including, Yoshida et al (2008) (46) and Piepkorn et al (2003) (47) found this gene to be overexpressed in inflammatory dermatitis conditions, such as psoriasis. Based upon node degree, LEP, was the most connected gene in the mild psoriasis group. In a meta-analysis by Zhu et al (2013) (48), LEP was found to be overexpressed in patients with psoriasis compared to healthy controls. Manczinger and Kemény (2013) (49), in their study, encoded LEP as a differentially expressed gene for psoriasis. The Cytohubba plugin identified hub genes among both psoriasis 
sub-types; PLIN1, TLR4, ADIPOQ, CXCL8, PDK4, CXCL1, CXCL5, LPL, AGT and LEP were identified as hub genes in mild psoriasis. It is interesting to note that $\mathrm{T}_{\mathrm{H}} 17$ (CXCL1 and CXCL5)- and $\mathrm{T}_{\mathrm{H}} 1$ (CXCL8)-regulated cytokines were identified as hub genes and even the gene expression patterns of these $T_{H} 17$ - and $T_{H} 1$-regulated cytokines in the mild psoriasis sub-type were upregulated in comparison to severe psoriasis. These cytokines can be considered as central players in the initiation of the disease. The previously available literature suggests the role of these identified hub genes (CXCL8, TLR4, LEP, LPL, AGT, CXCL1 and ADIPOQ) specifically in psoriasis vulgaris (50-55). Although, the roles of PDK4 and CXCL5 have been studied in psoriasis $(56,57)$, their specific roles have not been studied in psoriasis sub-types, psoriasis vulgaris, at least to the best of our knowledge. Moreover, the present study reported PLIN1, PDK4 and CXCL5 as hub genes among the mild psoriasis subtypes, which could be of interest for further evaluation.

Similarly, BUB1, CCNB1, CCNA2, CDK1, CDH1, VEGFA, PLK1, CDC42, CCND1 and CXCL8 were the top 10 hub genes. Among these, only CXCL8, CDH1, VEGFA, CCND1, CDK1 and CCNB1 have been reported in psoriasis vulgaris $(53,54,58-61)$. In addition to these CCNA2, BUB1 and PLK1 have also been known in psoriasis $(49,61,62)$. Furthermore, the present study, for the first time (to the best of our knowledge), reported CDC42 in psoriasis and its sub-type along with CCNA2, BUB1 and PLK1 in severe psoriasis vulgaris. The pathway analysis tool is useful for classifying the continually increasing literature that explores the molecular mechanisms of the pathogenesis of psoriasis and remission after treatment. To investigate further, the top 3 clusters obtained from both the psoriasis sub-types using MCODE were assessed. CXCL5, GPAM and SCGB2A2 were identified as seed genes in clusters of mild psoriasis. Notably, CXCL5 was identified as the hub of cluster 1 and was also identified as one of the top 10 hub genes using network analysis. Hence, it substantiates the present claim of CXCL5 being a core gene in the mild-psoriasis group. Furthermore, the pathway enrichment of these clusters (Table IA) revealed it to be mostly enriched in the rheumatoid arthritis (hsa05323), IL-17 signalling pathway (hsa04657), cytokine-cytokine receptor interaction (hsa04060), PPAR signaling pathway (hsa03320), Th1 and Th2 cell differentiation (hsa04658) and Th17 cell differentiation (hsa04659). Likewise, in severe psoriasis, CDKN3, TK1 and ATM were reported as the seed genes in the top 3 clusters, respectively. Based on the degree ranking of cluster 1, CCNA2, CDK1, BUB1 and CCNB1 were identified as cluster hub genes and additionally, these genes were initially reported as hub genes in the present network analysis of severe psoriasis vulgaris. The cell cycle pathway (hsa04110), chemokine signalling pathway (hsa04062) and cytokine-cytokine receptor interaction (hsa04060) were the most enriched pathways in the severe psoriasis group (Table IB). Overall, the pathway enrichment these clusters from both the groups provide ample evidence of psoriasis to be a disease impaired by the immune system.

Furthermore, the present study analysed the expression levels of top 2 hub genes (CDK1 and CDH1), based upon their presence in 12 built in parameters of the Cytohubba plug in psoriasis tissues using immunohistochemical analysis. The results revealed that CDK1 protein was overexpressed (positively intense stained) in psoriatic skin lesions as compared to non-lesional areas. This observation was consistent with that in the study by Greenberg et al (2020) (63), who reported an increased CDK1 expression in psoriatic lesions. The psoriasis skin lesions and non-lesional skins were assessed for the expression and localization of $\mathrm{CDH} 1$. The expression of $\mathrm{CDH} 1$ was spatially decreased in the epidermis of lesional psoriasis compared with non-lesional epidermis, specifically in the basal cell and upper granular layers of the epidermis. These findings are in contrast to the reported interpretation of $\mathrm{CDH} 1$ expression in psoriasis published by Zhou et al (2003) (64), whereas akin to what was reported in the earlier study by Chung et al (2005) (65). These results suggest that the evaluation of CDK1 and CDH1 may be a useful index for the prognosis of and may act as a therapeutic marker of psoriasis vulgaris.

The present study analysed the gene expression patterns in the moderate and severe psoriasis group. The top $2 \mathrm{hub}$ genes were validated using immunohistochemistry. However; the absence of another dataset in NCBI-GEO, including mild-severe psoriasis samples may be considered a setback of the present study. Additionally, further studies with larger sample sizes are required to uphold the persuasiveness of the reported results. The main highlighted DEGs need to be further experimentally validated by western blot analysis and RT-qPCR analysis to affirm their role as drug targets and potential biomarkers for psoriasis.

In conclusion, the present study demonstrates that the gene expression analysis identified several important DEGs that may play a central role in the initiation, development and prognosis of psoriasis. To the best of our knowledge, the present study is the first to report the involvement of the CDC42 gene in the psoriasis transcriptome. The study highlighted nearly 73 genes that could be validated for its progression from the mild to psoriasis disease state. Immunohistochemical analysis established that the downregulation of $\mathrm{CDH} 1$ and the upregulation of CDK1 in psoriasis lesions may be responsible for the stimulation of both psoriatic epidermal hyperproliferation, as well as psoriatic inflammation.

\section{Acknowledgements}

The authors acknowledge Ms. Noor Saba Khan and Ms. Pallavi Saxena for editing the images provided in the manuscript.

\section{Funding}

The study was supported by the ICMR-National Institute of Pathology and Indian Council of Medical Research [grant no. ISRM/12(05)/2017].

\section{Availability of data and materials}

The datasets used and/or analysed during the current study are available from the corresponding author on reasonable request.

\section{Authors' contributions}

SC, DP, GT and AKJ designed the study. SC, AKJ, GT and HS reviewed the literature. SC, RA, HS and DP conducted 
and interpreted the bioinformatics analysis. PP was the clinician involved in enrolling the patients and in diagnosing the psoriasis vulgaris cases. BB, SNK and SC performed the immunohistochemical analysis. BB, SNK and PP interpreted the results of immunohistochemistry. SC, DP and BB prepared the draft of the manuscript. All authors revised and approved the final manuscript.

\section{Ethics approval and consent to participate}

Ethical approval from the Ethics Committee of Vardhman Mahavir Medical College and Safdarjung Hospital, New Delhi (IEC/VMMC/SIH/Project/2018/1094) was obtained. Informed written consent was obtained from the patients.

\section{Patient consent for publication}

Not applicable.

\section{Competing interests}

The authors declare that they have no competing interests.

\section{References}

1. World Psoriasis Day: International Federation of Psoriasis Associations.

2. Singh S, Pradhan D, Puri P, Ramesh V, Aggarwal S, Nayek A and Jain AK: Genomic alterations driving psoriasis pathogenesis. Gene 683: 61-71, 2019.

3. Huang YH, Kuo CF, Huang LH and Hsieh MY: Familial aggregation of psoriasis and co-aggregation of autoimmune diseases in affected families. J Clin Med 8: 115, 2019.

4. Menter MA, Armstrong AW, Gordon KB and Wu JJ: Common and not-so-common comorbidities of psoriasis. Semin Cutan Med Surg 37: S48-S51, 2018.

5. Takeshita J, Grewal S, Langan SM, Mehta NN, Ogdie A, Van Voorhees AS and Gelfand JM: Psoriasis and comorbid diseases part I. Epidemiology. J Am Acad Dermatol 76: 377-390, 2017.

6. Choudhary S, Patel R, Pradhan D, Deval R, Singh H, Thomas G and Jain AK: Psoriasis and cardiovascular disorders: Association or epiphenomenon? Meta-Analysis of observational studies. 3 Biotech 10: 104, 2020.

7. Schmitt $J$ and Wozel G: The psoriasis area and severity index is the adequate criterion to define severity in chronic plaque-type psoriasis. Dermatology 210: 194-199, 2005.

8. Boehncke WH and Schön MP: Psoriasis. Lancet 386: 983-994, 2015.

9. Nestle FO, Kaplan DH and Barker J: Psoriasis. New Engl J Med 361: 496-509, 2009.

10. Bowcock AM, Shannon W, Du F, Duncan J, Cao K, Aftergut K, Catier J, Fernandez-Vina MA and Menter A: Insights into psoriasis and other inflammatory diseases from large-scale gene expression studies. Hum Mol Genet 10: 1793-1805, 2001.

11. Oestreicher JL, Walters IB, Kikuchi T, Gilleaudeau P, Surette J, Schwertschlag U, Dorner AJ, Krueger JG and Trepicchio WL: Molecular classification of psoriasis disease-associated genes through pharmacogenomic expression profiling. Pharmacogenomics J 1: 272-287, 2001.

12. Zhou X, Krueger JG, Kao MC, Lee E, Du F, Menter A, Wong WH and Bowcock AM: Novel mechanisms of T-cell and dendritic cell activation revealed by profiling of psoriasis on the 63,100-element oligonucleotide array. Physiol Genomics 13 . $69-78,2003$

13. Kulski JK, Kenworthy W, Bellgard M, Taplin R, Okamoto K, Oka A, Mabuchi T, Ozawa A, Tamiya G and Inoko H: Gene expression profiling of Japanese psoriatic skin reveals an increased activity in molecular stress and immune response signals. J Mol Med (Berl) 83: 964-975, 2005.

14. Mee JB, Johnson CM, Morar N, Burslem F and Groves RW: The psoriatic transcriptome closely resembles that induced by interleukin-1 in cultured keratinocytes. Am J Pathol 171: 32-42,2007.
15. Yao Y, Richman L, Morehouse C, de los Reyes M, Higgs BW, Boutrin A, White B, Coyle A, Krueger J, Kiener PA and Jallal B: Type I interferon: Potential therapeutic target for psoriasis? PLoS One 3: e2737, 2008.

16. Gudjonsson JE, Ding J, Johnston A, Tejasvi T, Guzman AM, Nair RP, Voorhees JJ, Abecasis GR and Elder JT: Assessment of the psoriatic transcriptome in a large sample: Additional regulated genes and comparisons with in vitro models. J Invest Dermatol 130: 1829-1840, 2010.

17. Choudhary S, Pradhan D, Khan NS, Singh H, Thomas G and Jain A: Decoding psoriasis: Integrated bioinformatics approach to understand hub genes and involved pathways. Curr Pharm Des 11: 10, 2020

18. Coda AB, Icen M, Smith JR and Sinha AA: Global transcriptional analysis of psoriatic skin and blood confirms known disease-associated pathways and highlights novel genomic 'hot spots' for differentially expressed genes. Genomics 100: 18-26, 2012.

19. Swindell WR, Johnston A, Voorhees JJ,Elder JT and Gudjonsson JE: Dissecting the psoriasis transcriptome: Inflammatory- and cytokine-driven gene expression in lesions from 163 patients. BMC Genomics 14: 527, 2013.

20. Kim J, Bissonnette R, Lee J, Correa da Rosa J, Suárez-Fariñas M, Lowes MA and Krueger JG: The spectrum of mild to severe psoriasis vulgaris is defined by a common activation of il-17 pathway genes, but with key differences in immune regulatory genes. J Invest Dermatol 136: 2173-2182, 2016.

21. Gautier L, Cope L, Bolstad BM and Irizarry RA: Affy-Analysis of affymetrix genechip data at the probe level. Bioinformatics 20: 307-315, 2004.

22. Sun C, Yuan Q, Wu D, Meng X and Wang B: Identification of core genes and outcome in gastric cancer using bioinformatics analysis. Oncotarget 8: 70271-70280, 2017.

23. Wang $\mathrm{Y}$ and Zheng T: Screening of hub genes and pathways in colorectal cancer with microarray technology. Pathol Oncol Res 20: 611-618, 2014.

24. Yu G, Wang LG, Han Y and He QY: ClusterProfiler: An R package for comparing biological themes among gene clusters. OMICS 16: 284-287, 2012.

25. Kanehisa M, Furumichi M, Tanabe M, Sato Y and Morishima K: KEGG: New perspectives on genomes, pathways, diseases and drugs. Nucleic Acids Res 45: D353-D361, 2017.

26. Welter D, MacArthur J, Morales J, Burdett T, Hall P, Junkins H, Klemm A, Flicek P, Manolio T, Hindorff L and Parkinson H: The NHGRI GWAS catalog, a curated resource of SNP-trait associations. Nucleic Acids Res 42: D1001-D1006, 2014.

27. Becker KG, Barnes KC, Bright TJ and Wang SA: The genetic association database. Nat Genet 36: 431-432, 2004.

28. Mattingly CJ, Rosenstein MC, Colby GT, Forrest JN Jr and Boyer JL: The comparative toxicogenomics database (CTD): A resource for comparative toxicological studies. J Exp Zool A Comp Exp Biol 305: 689-692, 2006.

29. Landrum MJ, Lee JM, Riley GR, Jang W, Rubinstein WS, Church DM and Maglott DR: ClinVar: Public archive of relationships among sequence variation and human phenotype. Nucleic Acids Res 42: D980-D985, 2014

30. Apweiler R, Bairoch A, Wu CH, Barker WC, Boeckmann B, Ferro S, Gasteiger E, Huang H, Lopez R, Magrane M, et al: UniProt: The universal protein knowledgebase. Nucleic Acids Res 32: D115-D119, 2004.

31. Piñero J, Bravo À, Queralt-Rosinach N, Gutiérrez-Sacristán A, Deu-Pons J, Centeno E, García-García J, Sanz F and Furlong LI: DisGeNET: A comprehensive platform integrating information on human disease-associated genes and variants. Nucleic Acids Res 45: D833-D839, 2017.

32. Carvalho-Silva D, Pierleoni A, Pignatelli M, Ong C, Fumis L, Karamanis N, Carmona M, Faulconbridge A, Hercules A, McAuley E, et al: Open targets platform: New developments and updates two years on. Nucleic Acids Res 47: D1056-D1065, 2019.

33. Szklarczyk D, Gable AL, Lyon D, Junge A, Wyder S, Huerta-Cepas J, Simonovic M, Doncheva NT, Morris JH, Bork P, et al: STRING v11: Protein-protein association networks with increased coverage, supporting functional discovery in genome-wide experimental datasets. Nucleic Acids Res 47: D607-D613, 2019.

34. Shannon P, Markiel A, Ozier O, Baliga NS, Wang JT, Ramage D, Amin N, Schwikowski B and Ideker T: Cytoscape: A software environment for integrated models of biomolecular interaction networks. Genome Res 13: 2498-2504, 2003. 
35. Yang Y, Zhong Z, Ding Y, Zhang W, Ma Y and Zhou L: Bioinformatic identification of key genes and pathways that may be involved in the pathogenesis of $\mathrm{HBV}$-associated acute liver failure. Genes Dis 5: 349-357, 2018.

36. Nayak S, Pradhan D, Singh H and Reddy MS: Computational screening of potential drug targets for pathogens causing bacterial pneumonia. Microb Pathog 130: 271-282, 2019.

37. Lowes MA, Suárez-Fariñas M and Krueger JG: Immunology of psoriasis. Annu Rev Immunol 32: 227-255, 2014.

38. Campanati A, Goteri G, Simonetti O, Ganzetti G, Giuliodori K, Stramazzotti D, Morichetti D, Bernardini ML, Mannello B, Fabris G and Offidani A: CTACK/CCL27 expression in psoriatic skin and its modification after administration of etanercept. Br J Dermatol 157: 1155-1160, 2007.

39. Gudjonsson J, Johnston A, Stoll S, Riblett MB, Xing X, Kochkodan JJ, Ding J, Nair RP, Aphale A, Voorhees JJ and Elder JT: Evidence for altered wnt signaling in psoriatic skin J Invest Dermatol 130: 1849-1859, 2010.

40. Wang X, Liu X, Liu N and Chen H: Prediction of crucial epigenetically-associated, differentially expressed genes by integrated bioinformatics analysis and the identification of S100A9 as a novel biomarker in psoriasis. Int J Mol Med 45: 93-102, 2020.

41. Ogawa E, Sato Y, Minagawa A and Okuyama R: Pathogenesis of psoriasis and development of treatment. J Dermatol 45: 264-272, 2018.

42. Chen W, Xie K, Liu X and Chen H: Identification of key pathways and genes in psoriasis via gene microarray analysis. Mol Med Rep 13: 2327-2337, 2016

43. Tan X, Zhang X, Pan L, Tian X and Dong P: Identification of key pathways and genes in advanced coronary atherosclerosis using bioinformatics analysis. Biomed Res Int 2017: e4323496, 2017.

44. Elfatoiki FZ, Azhari ME, Kettani AE, Serhier Z, Othmani MB, Timinouni M, Benchikhi H, Chiheb S and Fellah H: Psoriasis and staphylococcus aureus skin colonization in moroccan patients. Pan Afr Med J 23: 33, 2016.

45. Johnston A, Gudjonsson JE, Aphale A, Guzman AM, Stoll SW and Elder JT: EGFR and IL-1 signaling synergistically promote keratinocyte antimicrobial defenses in a differentiation-dependent manner. J Invest Dermatol 131: 329-337, 2011.

46. Yoshida A, Kanno H, Watabe D, Akasaka T and Sawai T: The role of heparin-binding EGF-like growth factor and amphiregulin in the epidermal proliferation of psoriasis in cooperation with TNFalpha. Arch Dermatol Res 300: 37-45, 2008.

47. Piepkorn $M$, Predd $H$, Underwood $R$ and Cook $P$. Proliferation-differentiation relationships in the expression of heparin-binding epidermal growth factor-related factors and erbB receptors by normal and psoriatic human keratinocytes Arch Dermatol Res 295: 93-101, 2003.

48. Zhu KJ, Zhang C, Li M, Zhu CY, Shi G and Fan YM: Leptin levels in patients with psoriasis: A meta-analysis. Clin Exp Dermatol 38: 478-483, 2013

49. Manczinger M and Kemény L: Novel factors in the pathogenesis of psoriasis and potential drug candidates are found with systems biology approach. PLoS One 8: e80751, 2013

50. Khabour OF, Alomari MA and Abu Obaid AA: The relationship of adiponectin level and ADIPOQ gene variants with BMI among young adult women. Dermatoendocrinol 10: e1477902, 2018.

51. Kyriakou A, Patsatsi A, Sotiriadis D and Goulis DG: Serum leptin, resistin, and adiponectin concentrations in psoriasis: A meta-analysis of observational studies. Dermatology 233: 378-389, 2017.
52. Campanati A, Consales V, Orciani M, Giuliodori K, Ganzetti G, Bobyr I, Sorgentoni G, di Primio R and Offidani A: Role of mesenchymal stem cells in the pathogenesis of psoriasis: Current perspectives. Psoriasis (Auckl) 7: 73-85, 2017.

53. Ayala-Fontánez N, Soler DC and McCormick TS: Current knowledge on psoriasis and autoimmune diseases. Psoriasis (Auckl) 6: 7-32, 2016.

54. Suárez-Fariñas M, Li K, Fuentes-Duculan J, Hayden K, Brodmerkel C and Krueger JG: Expanding the psoriasis disease profile: Interrogation of the skin and serum of patients with moderate-to-severe psoriasis. J Invest Dermatol 132: 2552-2564, 2012.

55. Pietrzak A, Michalak-Stoma A, Chodorowska G and Szepietowski JC: Lipid disturbances in psoriasis: An update. Mediators Inflamm 2010: 535612, 2010.

56. Disteldorf EM, Krebs CF, Paust HJ, Turner JE, Nouailles G, Tittel A, Meyer-Schwesinger C, Stege G, Brix S, Velden J, et al: CXCL5 drives neutrophil recruitment in TH17-mediated GN. J Am Soc Nephrol 26: 55-66, 2015.

57. Adhikary T, Wortmann A, Schumann T, Finkernagel F, Lieber S, Roth K, Toth PM, Diederich WE, Nist A, Stiewe T, et al: The transcriptional PPAR $\beta / \delta$ network in human macrophages defines a unique agonist-induced activation state. Nucleic Acids Res 43: 5033-5051, 2015

58. Gerkowicz A, Socha M, Pietrzak A, Zubilewicz T and Krasowska D: The role of VEGF in psoriasis: An update. Acta Angiologica 24: 134-140, 2018

59. Xue X, Wu J, Li J, Xu J, Dai H, Tao C, Li C and Hu J: Indirubin attenuates mouse psoriasis-like skin lesion in a CD274-dependent manner: An achievement of RNA sequencing. Biosci Rep 38: BSR20180958, 2018.

60. Korkmaz S and Korkmaz H: Effect of alterations in apoptotic pathway on development of metabolic syndrome in patients with psoriasis vulgaris. Br J Dermatol 176: 1549-1557, 2017.

61. Zaba LC, Suárez-Fariñas M, Fuentes-Duculan J, Nograles KE, Guttman-Yassky E, Cardinale I, Lowes MA and Krueger JG: Effective treatment of psoriasis with etanercept is linked to suppression of IL-17 signaling, not immediate response TNF genes. J Allergy Clin Immunol 124: 1022-1010, 2009.

62. Li GB, Ma S, Yang LL, Ji S, Fang Z, Zhang G, Wang LJ, Zhong JM, Xiong Y, Wang JH, et al: Drug discovery against psoriasis: Identification of a new potent fms-like tyrosine kinase 3 (FLT3) inhibitor, 1-(4-((1H-Pyrazolo[3,4-d]pyrimidin-4-yl)oxy)3-fluorophenyl)-3-(5-(tert-butyl)isoxazol-3-yl)urea, that showed potent activity in a psoriatic animal model. J Med Chem 59: 8293-8305, 2016.

63. Greenberg EN, Marshall ME, Jin S, Venkatesh S, Dragan M, Tsoi LC, Gudjonsson E, Nie Q, Takahashi JS and Andersen B: Circadian control of interferon-sensitive gene expression in murine skin. Proc Natl Acad Sci USA 117: 5761-5771, 2020.

64. Zhou S, Matsuyoshi N, Takeuchi T, Ohtsuki Y and Miyachi Y: Reciprocal altered expression of T-cadherin and P-cadherin in psoriasis vulgaris. Br J Dermatol 149: 268-273, 2003.

65. Chung E, Cook PW, Parkos CA, Park YK, Pittelkow MR and Coffey RJ: Amphiregulin causes functional downregulation of adherens junctions in psoriasis. J Invest Dermatol 124: 1134-1140, 2005 .

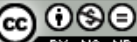

This work is licensed under a Creative Commons Attribution-NonCommercial-NoDerivatives 4.0 International (CC BY-NC-ND 4.0) License. 